\title{
Global context effects on musical expectancy
}

\author{
E. BIGAND and M. PINEAU \\ Université de Bourgogne, Dijon, France
}

\begin{abstract}
The effects of global harmonic contexts on expectancy formation were studied in a set of three experiments. Eight-chord sequences were presented to subjects. Expectations for the last chord were varied by manipulating the harmonic context created by the first six: in one context, the last chord was part of an authentic cadence (V-I), whereas in the other, it was a fourth harmonic degree following a full cadence (I-IV). Given this change in harmonic function, the last chord was assumed to be more expected in the former context, all the other local parameters being held constant. The effect of global context on expectancy formation was supported by the fact that subjects reported a lower degree of completion for sequences ending on an unexpected chord (Experiment 1), took longer to decide whether the last chord belonged to the sequence when the last chord was unexpected (Experiment 2), and took longer to decide whether the last chord was consonant or dissonant when it was unexpected (Experiment 3). These results are discussed with reference to current models of tonal cognition.
\end{abstract}

Musical expectancy is a compelling concept for investigation, for it plays a central role in both music theory (Lerdahl \& Jackendoff, 1983; L. B. Meyer, 1956, 1973; Narmour, 1990; Schenker, 1935, 1979) and cognitive psychology (Jones, 1976, 1987; Jones \& Yee, 1993). During the last decade, it has been shown that musical expectancy is governed by several features, including melodic interval size and melodic contour (Boltz \& Jones, 1986; Carlsen, 1981; Cuddy \& Lunney, 1995; Krumhansl, 1995; Schellenberg, 1996; Unyk \& Carlsen, 1987), rhythm (Boltz, 1993; Jones, Boltz, \& Kidd, 1982; Jones, Boltz, \& Klein, 1993; Schmuckler, 1990), and tonal-harmonic hierarchy (Bharucha \& Stoeckig, 1986, 1987; Schmuckler, 1989; Schmuckler \& Boltz, 1994). Some of these features (melodic contour and melodic interval size, for example) reflect the influence of general principles of perceptual organization; others (like the tonal-harmonic hierarchy) reflect the importance of the listeners' knowledge of a given musical idiom. The present study focuses on the musical expectancies derived from knowledge of the tonal-harmonic hierarchy. The main purpose was to investigate the effect of global harmonic context on expectancy formation.

\section{Tonal-Harmonic Hierarchy}

In the Western musical system, 12 notes of the chromatic scale are organized into several subsets of 7 notes, called diatonic scales. In each scale, seven diatonic chords are possible, each built on a different degree of the scale. Chords built on the first, the fifth, and the fourth scale degrees (referred to as tonic, dominant, and subdominant

This research was supported by research grants from the G.I.S. "Sciences de la Cognition" (Affectivité \& Cognition). We would like to thank N. Macmillan. M. A. Schmuckler, and B. Tillmann for their insightful comments. Correspondence concerning this article should be addressed to E. Bigand, LEAD-CNRS, 6, Boulevard Gabirel, Faculté des Sciences, F-21000 Dijon, France (e-mail: bigand@ satie.u-bourgogne.fr). chords, respectively) usually have a more central syntactic function than do those built on the other scale degrees. This creates a within-key hierarchy.

A fundamental aspect of the Western tonal system is that chords (and notes) belong to several keys: for example, the $C$ chord belongs to the $C, F$, and $G$ major keys, and to the $\mathrm{A}$ and $\mathrm{E}$ minor keys. Keys sharing the same chords and chords that share parent keys are said to be harmonically related (such as, e.g., the chords $\mathrm{C}$ and $\mathrm{B} b$ which both belong to the key of F). Since chords belong to several keys, their function varies with the context in which they appear: a $\mathrm{C}$ chord functions as a stable tonic chord in a $\mathrm{C}$ major context and as less stable dominant and subdominant chord in the $F$ and $G$ major key contexts, respectively. Understanding the change in function of the musical events with respect to the musical context is crucial to understanding Western musical pieces.

A number of experimental studies have shown that listeners have internalized the main aspects of the tonalharmonic hierarchy. According to Krumhansl (1990), once the key of a musical context is recognized, the tonic chord (I) is perceived as more stable than the dominant chord (V) and the subdominant chord (IV), which in turn are perceived as more stable than other diatonic and nondiatonic chords. Changing the key of the context leads to changes in the chord's function, so that a chord that was perceived as stable in a given context may become unstable in another key context and vice versa (Bharucha \& Krumhansl, 1983).

\section{Harmonic Expectancy}

The connectionist model developed by Bharucha (1987) provides an elegant framework for understanding how the knowledge of the tonal hierarchy may govern harmonic expectancies in listeners. In this model, the 12 tones of the chromatic scale are linked to major and minor chords, which are linked to major and minor keys. Tonal hierarchies are represented by the strength of the con- 
nections that link tone units to chords and key units. When a chord is sounded, activation spreads through the network via connected links, so that the key in which the chord is a very stable event receives the stronger activation. The harmonic expectations generated by a previous musical context are instantiated by the amount of activation that reverberates from key units to chord units. To take an example, when the $\mathrm{C}$ chord is sounded, it strongly activates the $C$ key unit, and to a lesser extent the $G$ and $F$ key units, in which it functions as the dominant and subdominant chords, respectively (see Bharucha, 1987). This activation then reverberates back to chord units belonging to these keys, so that some chords harmonically related to the $\mathrm{C}$ chord (i.e., F, G, and, to a lesser extent, $\mathrm{B}$ and $D$ ), receive stronger activation than others (e.g., $F \#$ or B). Therefore, the stronger the activation of one chord unit, the more expected the chord. In other words, the model would expect an $\mathrm{F}$ or $\mathrm{G}$ chord after the $\mathrm{C}$ chord, but not an $\mathrm{F} \#$ or $\mathrm{B}$ chord.

Empirical studies using a priming paradigm provide strong support for Bharucha's model of harmonic expectation. The main assumption was that a previous chord would prime the harmonically related chords so that the processing of these chords would be speeded up. To test this hypothesis, Bharucha and Stoeckig $(1986,1987)$ and Tekman and Bharucha (1992) asked subjects to decide as quickly as possible whether a target chord following a prime chord was in tune. The priming effect was shown by (1) a bias to judge targets to be in tune when they were related to the prime and out of tune when they were unrelated to it, and (2) shorter response times for in-tune targets when they were related to the prime, and for out-of-tune target when they were unrelated to it. According to the authors, a previous musical context (one chord in these experiments) generates expectancies for related chords to follow, resulting in greater consonance and faster processing for expected chords. Findings consistent with these were recently reported by Schmuckler and Boltz (1994), who used relatively long musical sequences as primes. The degree of harmonic expectancy for the last chord was varied from high to medium to low with chords of low expectancy as nondiatonic chords. Among other findings, it appeared that the chords whose harmonic relationship to the previous sequence was weak were rated as weakly expected (Experiment 1) and were not considered as "belonging" to the sequence, and that the response time to provide the correct "belong" or "not belong" response varied systematically as a function of the harmonic relatedness of the last chord (Experiment 2).

\section{Effects of Global or Local Harmonic Context on Expectancy Formation?}

Studies by Bharucha and Stoeckig $(1986,1987)$ and Tekman and Bharucha (1992) have provided evidence that one chord can prime another when both are harmonically related. However, since the prime and the target chords were played without any musical context, these studies did not address the eventual role played by higher level harmonic structure in harmonic expectancy forma- tion. Schmuckler and Boltz (1994) used a longer harmonic context as prime. However, since the harmonic expectation for the last chord was varied by using different final events, their study did not disentangle the potential influence of global and local structures. An important issue, therefore, is to pursue the investigation of whether harmonic expectancies occur at a very local level (i.e., between successive chords) or also at higher levels of musical structure.

In cognitive theories of music, the importance of higher level structures was emphasized by L. B. Meyer (1973) and Lerdahl and Jackendoff (1983), while more recent works have focused on the role played by local structures (Narmour, 1989, 1990; see also Cuddy \& Lunney, 1995; Krumhansl, 1995; Schellenberg, 1996). In cognitive psychology, the influence of global versus local context has been widely investigated in studies of language comprehension. Previous findings of D. E. Meyer and his associates (D. E. Meyer \& Schvaneveldt, 1971; D. E. Meyer, Schvaneveldt, \& Ruddy, 1974) have provided evidence for local priming between semantically related words. However, several recent experiments have shown a strong effect of the global semantic contexts on lexical processing (Foss, 1982; Foss \& Ross, 1983; Foss \& Speer, 1991; Hess, Foss, \& Carroll, 1995). In these studies, the relationship between a critical word and the general topic of the discourse (global context) was crossed with the relationship between the critical word and the word prior to it (local context). The main outcome was that facilitation of a target word depends on whether or not the global context is related to the target, regardless of the local context.

In the present study, our first purpose was to investigate the effect of global harmonic contexts on harmonic expectancies. In the following experiments, we manipulated the global harmonic context in order to change the harmonic relation between a target chord and the global context, while holding the local context constant (i.e., the chord prior to the target chord). If harmonic expectancies occur at a high level of musical structure, the target chord would be expected in one context but not in the other, and its processing would, as a result, be facilitated.

\section{Cognitive or Sensory Components in Harmonic Expectancy?}

Manipulating the global context also permits the further investigation of the cognitive versus sensory component underlying the formation of harmonic expectancies. In language, related prime-target pairs are related in meaning but not acoustically. Therefore, semantic priming is obviously based on a cognitive component. In music, priming effects do not necessarily imply the activation of abstract knowledge. According to Schmuckler (1989), "a chord sharing component tones, or overtones, with a preceding chord will be more highly anticipated than a continuation containing no overlapping frequencies with its predecessor" (p. 134). Therefore, an increase in processing time for an unexpected chord may be provoked by a surprising discontinuity at the very peripheral level. In Western tonal music, this issue is all the more crucial, 
since theoretical accounts of harmonic hierarchy and psychoacoustical accounts of chordal dissonance are, to a large extent, intrinsically related (see Bigand, Parncutt, \& Lerdahl, 1996; Krumhansl, 1990; Parncutt, 1989).

In Bharucha and Stoeckig's (1986) studies, the different harmonic relations between the primes and the targets were instantiated via different chords, so that changes in harmonic function were confounded with change in acoustical features. In order to remedy this problem, Bharucha and Stoeckig (1987, Experiment 1) used prime and target chords that do not share pitches and whose component tones contained only the harmonics 1, 2, and 4 (Experiment 2). Both experiments supported priming effects, suggesting that harmonic expectancies involve a cognitive component. In further experiments, Tekman and Bharucha (1992) observed that the priming effect did not diminish when the stimulus onset asynchrony between the prime and the target was as long as $2,500 \mathrm{msec}$ nor when a noise mask was inserted after the prime.

These results provide evidence that harmonic priming occurs at a cognitive level of representation. However, this conclusion should be moderated for several reasons. First, avoiding the overlap in harmonic frequency spectra does not definitely eliminate the differences in melodic arrangement that exist between the tones of the priming chord and those of both kinds of target. Melodic features play an important role in the perceived stability of chords (Bigand et al., 1996) and may well influence priming effects in music. Second, reducing the complexity of the harmonic spectra meant using sounds that differed greatly from those of musical instruments: with complex musical sounds, a sensory component may prevail over a cognitive one, because the amount of available sensory information is greater. Finally, in all these studies, priming effects were observed when very different harmonic relations were compared. Related targets (e.g., a Bb chord) are very close to the prime ( $\mathrm{C}$ chord), since both belong to the same key ( $F$ major) and function as strong reference points in this key. Unrelated targets $\left(F^{*}\right)$ are very far from the prime $(\mathrm{C})$, since to establish the shortest harmonic distance between them it is necessary to analyze the former as the dominant chord belonging to the third degree of the dominant key of the prime (i.e., C:V/iii/V; see Bigand et al., 1996). A cognitive component may actually govern harmonic expectations, but only when very different harmonic relations are compared (see Schmuckler \& Boltz, 1994, for consistent findings).

Our second purpose in the present study was to remedy these problems. Musical stimuli were played with piano-like sounds, and the harmonic relation between the target chord and the prime varied from tonic to subdominant. In order to avoid sensory priming, the target chords were preceded by a chord that was unchanged across the experimental situations. In the three experiments reported here, the target chord ended an eightchord sequence. For half of the sequences, the target chord functioned as a stable tonic chord and was assumed to be expected (Figure 1, left). The degree of expectancy for the target chord was varied in the other half of the sequences by manipulating the first six chords while never altering the last two (Figure 1, right). In these cases, the target functioned as a less stable subdominant chord following a cadence and was assumed to be less expected. This hypothesis was tested with three different tasks commonly used in musical expectancy studies. In Experiment 1, subjects were asked to perform a phrase completion judgment (Boltz, 1989a, 1989b); in Experiment 2, to make speeded reaction time judgments on whether the final chord belonged to the previous context (Schmuckler \& Boltz, 1994); and in Experiment 3, to make speeded reaction time judgments of whether the final chord was consonant or dissonant (Bharucha \& Stoeckig, 1986, 1987; Tekman \& Bharucha, 1992). In Experiments 1 and 2 , the subjects had no musical training. The influence of musical expertise was investigated in the last experiment.

\section{EXPERIMENT 1 Phrase Completion Judgments}

\section{Method}

Subjects. Fifteen students volunteers from an introductory course at the University of Dijon participated in this experiment. None had ever received formal musical training or learned a musical instrument.

Apparatus. All stimuli were played with sampled piano sounds produced by the EMT 10 Yamaha Sound Expander at a tempo of 60 quarter notes per minute, and without rubato. The Yamaha sampler was controlled through a MIDI interface by a Macintosh computer running Performer software. Velocity was constant for all the pitches, and there was no silence between offsets and onsets of successive chords. The sound stimuli were captured by SoundEditPro software at $\mathrm{CD}$ quality ( 16 bits and $44 \mathrm{kHz}$ ). The experiment was run on PsyScope software (Cohen, MacWhinney, Flatt, \& Provost, 1993).

Material. Four chord sequences were used: three are reductions from chorales of J. S. Bach, the fourth from a chorale written by a French music teacher. All of them contained eight chords and were ended by a full cadence (V-I) (Figure 1, left). The first six chords of these sequences were systematically varied so that the four new sequences were in the dominant key (Figure 1, right). As far as possible, the melodic contour of the outer voices (i.e., soprano and bass voices) remained unchanged, and the changes in pitch were minimal so that the registers remained similar. The two last chords were acoustically identical, but their harmonic functions changed with the context: in one context, the last chord functioned as a tonic chord (I) as part of a full cadence (V-I), and in the other context, as a subdominant chord (IV) following a full cadence (Figure 1).

Procedure and Design. The subjects were asked to rate the degree of completion of each sequence on a 9-point scale $(1=$ very low; $9=$ very high $)$. After each sequence, the 9-point scale appeared on the computer screen, and they responded by pushing a numeric key on the key board. The computer recorded their judgments on each trial. The eight-chord sequences were played twice, in a random order for each subject.

\section{Results}

The average ratings of completion are displayed in Figure 2 . On the average, the degrees of completion were higher when the last chord was a tonic chord. A 2 (harmonic context) $\times 4$ (chord sequences) $\times 2$ (replication) analysis of variance (ANOVA) revealed a significant effect of harmonic context $[F(1,14)=165.63, p<.001]$ 

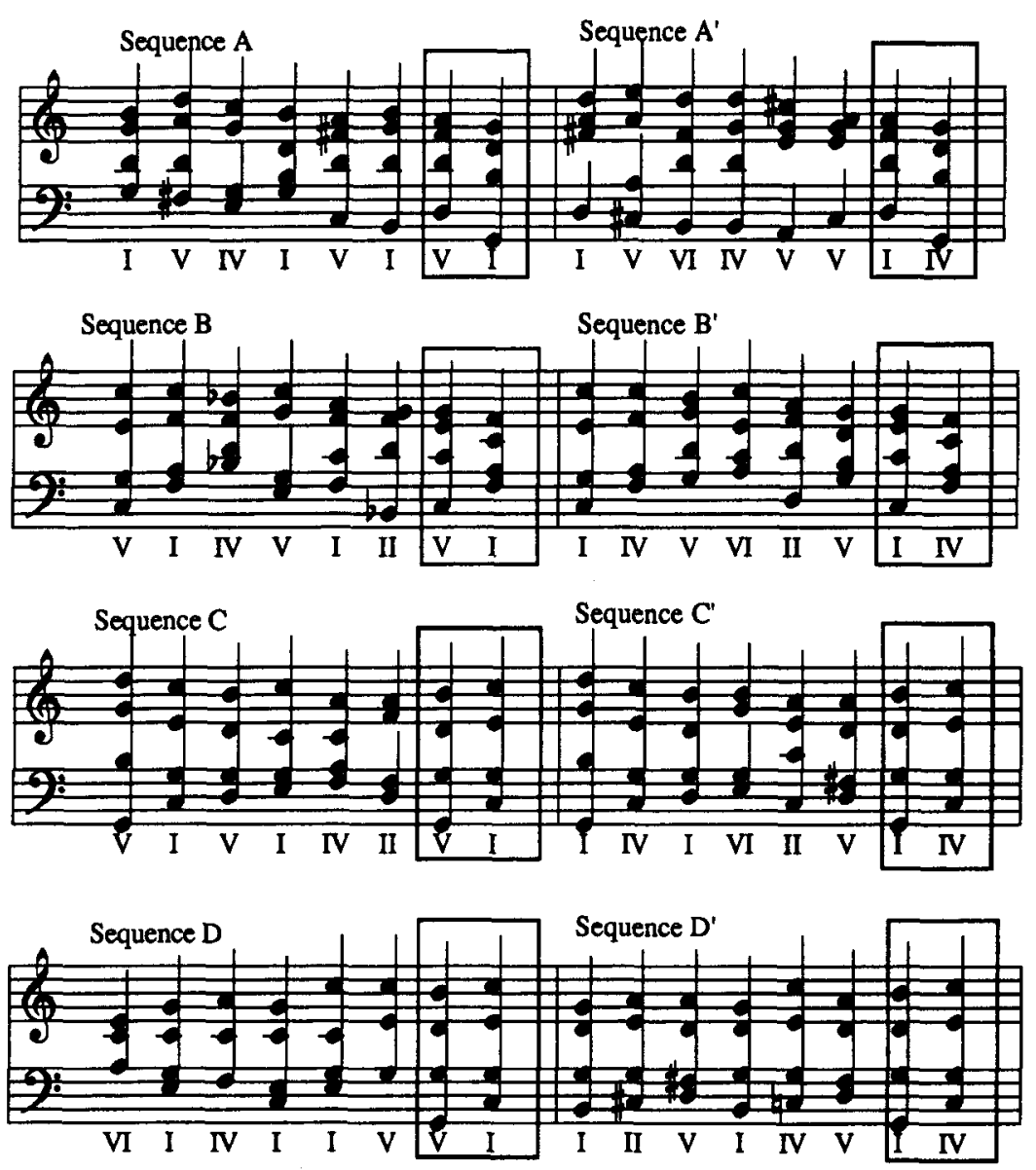

Figure 1. Chord sequences used in the experiments. Chord sequences $A, B$, and $D$ are reductions from the following Bach chorales: Freu's dich sehr, o meine Seele (A); Herr Jesu Christ, mein's Lebens Licht (B); Was mein Gott will, das g'scheh' allzeit (D).

and a significant second order interaction $[F(3,42)=$ $3.22, p<.04]$ : the effect of the harmonic context tended to vary with both the chord sequence and the number of times that subjects had heard a given sequence.

\section{Discussion}

This experiment demonstrates that the degree of completion experienced at the end of the sequences did not depend on the local harmonic and acoustic features of the two last chords: the sequences were perceived as more complete when the last chord functioned as a tonic chord than when it functioned as a subdominant chord. These results are consistent with several findings on melodic expectancies: listeners expected a harmonically stable event to end a musical sequence (Boltz, 1989a, 1989b, 1993; Jones et al., 1993; Palmer \& Krumhansl, 1987a, 1987b). They also confirmed the internalization of subtle aspects of the tonal-harmonic hierarchy by nonmusicians (Bigand, 1997; Bigand et al., 1996; Bharucha \& Krumhansl, 1983; Schmuckler, 1989). More importantly, they provided the first evidence that the global harmonic context governs harmonic expectancy.
It might be argued that the completion judgments were not necessarily caused by the changes in the harmonic relationships between the last chord and the global context. Despite the fact that the penultimate chord was kept constant, the judgments of completion could have been influenced by sensory priming instilled by the first six chords. The component frequencies of each tone of each chord might be accumulated in the auditory sensory memory until the last chord is perceived. The degree of completion experienced by the listeners could then depend on the number of overtones that the last chord shared with previous chords.

Further analysis was performed in order to test for possible global sensory priming. For each sequence, R. Parncutt computed the pitch commonality values between the last chord and all the previous ones (see Parncutt, 1989). These pitch commonality values were weighted in the present study by a coefficient of one for the first chord, two for the second, and so on until the penultimate chord, which received a coefficient of seven. For each sequence, these weighted pitch commonality values were averaged and the mean values were entered in a multiple regression 


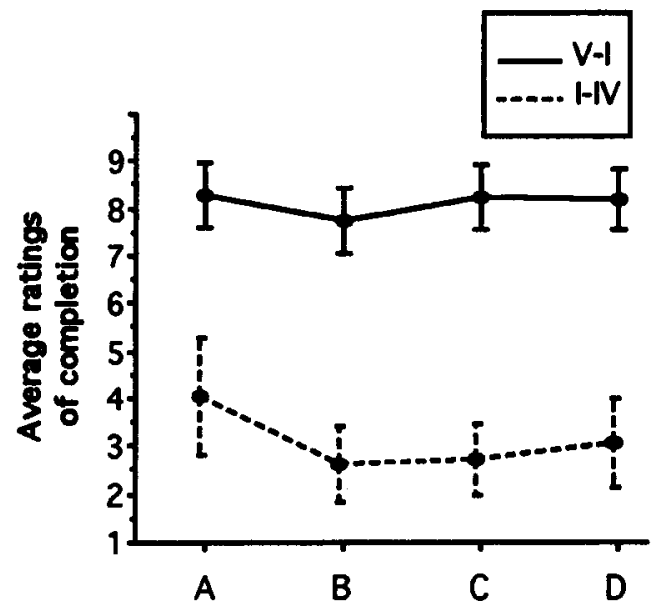

Figure 2. The $95 \%$ confidence intervals of average ratings for completion observed for Chord Sequences $A, B, C$, and D, when the last chord was expected (V-I) and unexpected (I-IV).

analysis. Following a reviewer's suggestion, the frequency and the time of occurrence of the last chord were also computed for each context. The harmonic function of the last chord was coded with a dummy variable. A hierarchical regression analysis was run to predict the subjects' ratings from a linear combination of these four variables. When the set of variables that expressed the surface properties was entered first in the model, we found that $R^{2}=.58[F(3,116)=54.66, p<.001]$. When the harmonic function of the last chord was added to the model, we found that $R^{2}=.77[F(4,115)=95.84, p<$ $.001]$. The highest semipartial correlation was observed for the harmonic function. This suggests that the effect of global context occurred mainly at a cognitive level of representation.

In Experiment 1, the task might have encouraged the subjects to consider the overall information of the musical sequences and not the specific information provided by the last chord. The purpose of Experiment 2 was to investigate the influence of the global context on the processing of the target chord. Following Schmuckler and Boltz (1994), subjects had to indicate as quickly as possible whether the last chord belonged to the chord sequence or was inserted by mistake. Since the last two chords remained acoustically unchanged in each condition, a difference in subjects' responses as a function of the harmonic context would indicate that the subjects differentiated between the structural functions of the last chord: "belong" responses should be more numerous when the target chord functions as a stable reference point (correct response) than when it functions as a less stable subdominant chord (false response). The main assumption concerned the response time: differences in response time as a function of the context would indicate that the processing of the harmonic function takes longer when the chord is not expected by the listeners.

\section{EXPERIMENT 2 "Belong"/“Not Belong" Judgments}

\begin{abstract}
Method
Subjects. Fifteen subjects, all studying psychology at the University of Dijon, participated in this experiment. None had ever received formal musical training or learned a musical instrument.

Material and Apparatus. The material and apparatus were identical to those described for Experiment 1, except that the time responses were registered here with the PsyScope button box clock, which permits a time accuracy of $2 \mathrm{msec}$. The clock started at the very beginning of the last chord and ended when subjects pushed the key.

Procedure and Design. The subjects were asked to decide as quickly as possible whether or not the last chord of each sequence sounded appropriate and seemed to belong with the preceding ones. They were informed that all the sequences contained eight chords and were allowed to respond even if the last chord of the sequence was still playing. The eight chord sequences were played twice in a random order for each subject.
\end{abstract}

\section{Results}

The first dependent measure involves the "belong"/ "not belong" responses. Tonic chords received a higher percentage of "belong" ratings ( $99 \%$ on the average) than did the subdominant chords $(18 \%)$. A 2 (harmonic context) $\times 4$ (chord sequences) $\times 2$ (replication) ANOVA revealed that manipulating the harmonic function of the final chord produced a significant variation in whether the subjects rated this final chord as belonging to the sequence $[F(1,14)=295.35, p<.001]$. With the use of a different task, Experiment 2 replicated the context effects observed in Experiment 1 for completion judgments. A further analysis provided evidence that completion and "belong" judgments were probably two strongly related measures of the same phenomenon: indeed the average degrees of completion were very highly correlated with the percentages of "belong" responses $[r(6)=.99, p<.01]$.

The second dependent measure involves response times (for both "belong" and "not belong" responses). As is illustrated in Figure 3, response times were $375 \mathrm{msec}$ shorter on the average for sequences ending on the tonic chord. A 2 (harmonic context) $\times 4$ (chord sequences) $\times$ 2 (replication) ANOVA confirmed that the processing time needed in order for subjects to respond was shorter for chords assumed to be expected $[F(1,14)=10.23$, $p<.01]$. A second analysis splitting the subjects' responses into correct and incorrect responses was also performed: "belong" judgments for sequences ending on the most stable chord (i.e., the tonic chord) were considered as correct responses. "Not belong" judgments were considered as correct for sequences ending on the more unstable subdominant chord. Only the response times for correct responses were considered (i.e., $91 \%$ of the number of total responses). Response times for correct responses were on the average $370 \mathrm{msec}$ shorter for sequences ending on the tonic chord $[F(1,14)=10.01, p<.01]$.

In order to test the influence of acoustic and cognitive factors, a hierarchic regression analysis was performed 


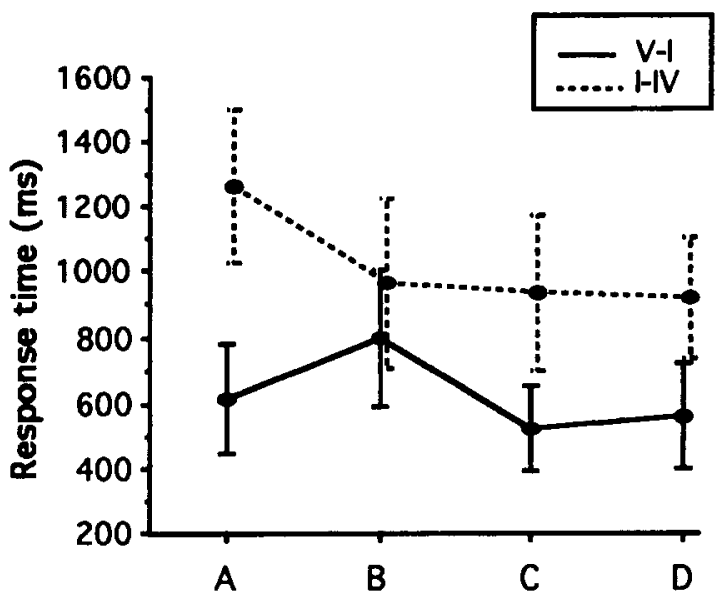

Figure 3. The $95 \%$ confidence intervals of average response time observed for Chord Sequences $A, B, C$, and $D$, when the target chord was expected (V-I) and unexpected (I-IV).

to predict the subjects' time responses from the averaged pitch commonality values, the frequency and the time of occurrence of the last chord, and the harmonic function of the last chord. When the first three variables were entered in the model, we found that $R^{2}=.296[F(3,116)=$ $16.22, p<.001]$. When the harmonic function was added, we found that $R^{2}=.347[F(4,115)=15.29, p<.001]$. When the four variables were entered in the regression model, the harmonic function was the sole variable that made a direct contribution to response times $(t=3.01$, $p<.01$ ).

\section{Discussion}

Since the present experimental task emphasized the importance of local context, we might have expected the global context to have a weaker or no effect. The present findings confirmed the effect of global context observed in Experiment 1: the last chord was more often perceived as belonging to the sequence when it functioned as a tonic chord. The effect of global context on harmonic expectancy found expression in the fact that response times (for all responses as for correct answers) were shorter for chord sequences ending on the more expected tonic chord. This suggests that the processing of the chord function is shorter for highly expected chords. This conclusion agrees with that of Schmuckler and Boltz (1994): events weakly related to a previous musical context take longer for listeners to analyze. In addition, the results of the regression analysis provided further evidence that this global priming effect was unlikely to occur at a sensory level.

In Experiments 1 and 2, we used subjective tasks. Our first purpose in Experiment 3 was to test the effect of the global context with a priming task similar to that used by Bharucha and Stoeckig $(1986,1987)$. According to those authors, a musical prime generates the expectancy that harmonically related chords will follow, resulting in greater consonance and faster processing for related tar- gets. It was, therefore, assumed that a consonant/dissonant decision concerning the last chord would be easier and shorter for expected target chords.

Our second purpose in Experiment 3 was to investigate the influence of musical expertise. Experiments 1 and 2 indicated that neither an explicit knowledge of the Western harmonic hierarchy nor extensive practice of music are indispensable for one to perceive small changes in chord function. Nevertheless, it should be assumed that the effect of context would be stronger with musicians.

\section{EXPERIMENT 3 “Consonant"/"Dissonant" Judgments}

\section{Method}

Subjects. Thirty listeners participated in this experiment. Fifteen were studying psychology (they are referred to below as nonmusicians) and had never received formal musical training, nor had they learned to play a musical instrument. Fifteen were students at the Music Conservatory of Dijon and had been studying music for at least 12 years. They had studied music theory and received ear training, and they had learned how to play a musical instrument (they are referred to below as musicians).

Material. The same chord sequences were used. For each of them, the last chord was either consonant or dissonant. Preliminary experiments indicated that nonmusicians had a lot of difficulty detecting subtle dissonances. Since we wanted to minimize the percentage of errors, the dissonances were defined in a way that was quite salient for nonmusicians. Target chords were rendered dissonant by increasing the pitch of the fifth by a semitone. In half of the cases, this dissonance was rendered more salient by increasing the amplitude of the augmented fifth by $25 \%$. In the other half, the amplitude of the augmented fifth was the same as that of the other tones. These changes in amplitude were also present when the last chords were consonant

Procedure. The experimental procedure was split into two phases. During the first phase, the subjects were trained to differentiate between dissonant and consonant chords. Sixteen chords were played in isolation and in a random order, and the subjects had to make a consonant/dissonant judgment as quickly as possible. The subjects were informed by a feedback sound signal whenever their responses were incorrect. The 16 training chords were constructed in a way similar to the experimental target chords: half were dissonant, half consonant. In half of the chords, the fifth was played $25 \%$ louder, and in the other half, it was played at the same level of intensity as were the other notes. Only the subjects who made fewer than five errors were allowed to continue the experiment. The others were run again in the training session.

During the second phase, the subjects were asked to perform a speeded reaction time judgment for the last chord of the sequences. They were informed that all the sequences contained eight chords, and that half of the sequences would finish with a dissonant chord, half with a consonant one. A feedback signal was sounded when the subjects answered incorrectly. The experiment was run with PsyScope software (see Experiment 2).

Design. Crossing the manipulations of harmonic context (two levels), dissonance (two levels), and intensity of the fifth (two levels) produced eight possible versions for each of the four chorales, yielding a total of 32 experimental patterns to be tested with each group of listeners. Each subject heard all 32 sequences, presented in a random order.

\section{Results}

The percentages of correct responses averaged across the two levels of loudness and the four chorales are dis- 
played in Figure 4. Because the dissonances were salient, these percentages were very high for both groups of subjects. A 2 (musical expertise) $\times 2$ (harmonic context) $\times$ 4 (chord sequences) $\times 2$ (consonance) $\times 2$ (loudness) ANOVA revealed that percentages of correct responses were higher for expected tonic chords $[F(1,28)=8.96$, $p<.01]$. The effect of musical expertise was marginally significant, with higher percentages for musicians $[F(1,28)=3.68, p<.07]$. As with the findings of Bharucha and Stoeckig $(1986,1987)$, the present experiment confirmed that consonant/dissonant judgments were more accurate for harmonically related targets. Bharucha and Stoeckig $(1986,1987)$ also observed a significant bias toward judging target chords to be consonant when expected and dissonant when unexpected. As is shown in Figure 4 , a consistent harmonic context $\times$ consonance interaction tended to occur with musicians only, but it failed to reach a significant level $(p=.11)$. The weakness of this interaction in the present experiment may be explained by the extent of the mistuning (i.e., the fifth was augmented here by a semitone, whereas it was lowered by an eighth tone in Bharucha and Stoeckig, 1987), and by the fact that the difference in harmonic relationships was reduced (see above). It is noteworthy that this interaction has not always been replicated by Tekman and Bharucha (1992, Experiments 3-4).

The second dependent measure involved the reaction time necessary for "consonant"/"dissonant" judgments. Response times were $174 \mathrm{msec}$ shorter, on the average, for expected tonic chords (Figure 5). Dissonant chords were detected, on the average, $229 \mathrm{msec}$ faster than consonant ones. As observed by Bharucha and Stoeckig (1987), the effect of the dissonance depended on the harmonic relationship of the target. Dissonant chords speeded up response times and to a larger extent than for unex-

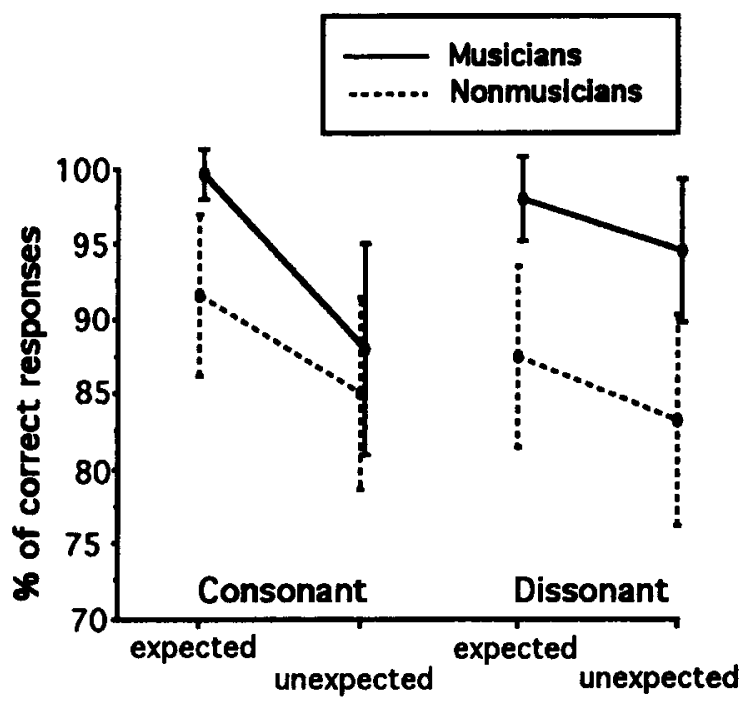

Figure 4. The $95 \%$ confidence intervals of average percent correct responses for consonant and dissonant targets observed when the target chord was expected (V-I) and unexpected (I-IV).

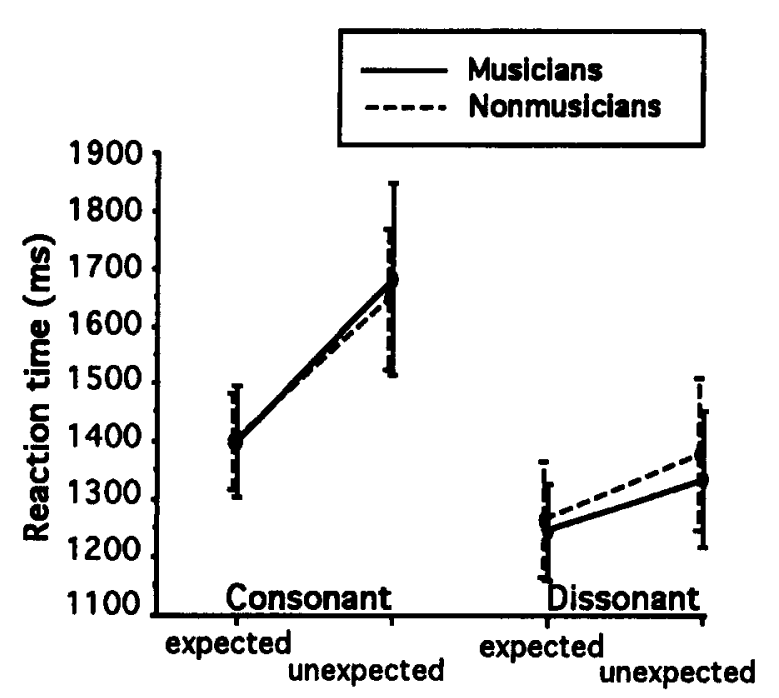

Figure 5. The $95 \%$ confidence intervals of average response time observed when the target chord was expected $(V-I)$ and unexpected (I-IV).

pected chords. The present interaction is consistent with the crossed interaction observed by Bharucha and Stoeckig (1987). A 2 (musical expertise) $\times 2$ (harmonic context) $\times 4$ (chord sequences) $\times 2$ (consonance) $\times 2$ (loudness) ANOVA indicated that the effect of the harmonic context was significant $[F(1,28)=23.08, p<.001]$, as was the effect of consonance $[F(1,28)=38.70, p<.001]$, and the interaction of harmonic context $x$ consonance $[F(1,28)=4.97, p<.05]$. In addition, there was also a significant consonance $\times$ intensity interaction, indicating that intensified fifths speeded up response times, but only for dissonant chords $[F(1,28)=6.95, p<.05]$. No main effect of musical expertise was observed, but there was a significant second order interaction: when the fifth was played at high amplitude, the effect of the harmonic context increased for the musicians but decreased for the nonmusicians $[F(1,28)=8.07, p<.01]$. A second analysis was also performed with the reaction time for correct responses as the dependent measure. Not surprisingly, this analysis confirmed the previous findings: reaction times were $156 \mathrm{msec}$ shorter, on the average, for expected tonic chords $[F(1,28)=26.19, p<.001]$ and $182 \mathrm{msec}$ shorter, on the average, for dissonant chords $[F(1,28)=$ $15.00, p<.001]$. There was no significant effect of musical expertise, but there was a significant musical expertise $\times$ harmonic context $\times$ intensity interaction $[F(1,28)=$ $6.123, p<.02]$.

In order to test the possible influence of acoustic factors on response times, a hierarchical regression analysis was performed to predict the subjects' time responses from the averaged pitch commonality values, the frequency and the time of occurrence of the last chord, and the harmonic function of the last chord. For each subject, response times were averaged across consonance and loudness conditions. For the two groups of subjects together, we found that $R^{2}=.14[F(3,236)=8.89, p<.001]$ 
when the first three variables were entered in the model. We found that $R^{2}=.24[F(4,235)=11.93, p<.001]$ when the harmonic function was added. When the four variables were entered into the regression model, the harmonic function was the sole variable that made a direct contribution to response time $(t=4.36, p<.01)$. Hierarchical analysis performed for each group separately led to the same results.

Finally, a further analysis of the nonmusicians' data revealed that mean reaction times for "consonant"/ "dissonant" judgments were correlated with mean response times for "belong"/"not belong" judgments $[r(6)=$ $.82, p<.05]$. This suggests that whatever the perceptual judgment required, an expected chord tends to be processed more quickly than a less expected one.

\section{Discussion}

The present findings indicate priming effects similar to those reported by Bharucha and colleagues (Bharucha \& Stoeckig, 1986, 1987; Tekman \& Bharucha, 1992). Target chords were more correctly and more quickly identified when they were harmonically related to the previous context. According to the authors, such a facilitation can be viewed as a robust demonstration of the anticipatory processing that underlies musical expectancies: expected chords were processed more easily and more quickly because they were activated by the context and were then partly processed before they actually sounded.

In comparison with previous studies, Experiment 3 indicates that the priming effect occurs for target chords whose harmonic relationships to the prime differ only slightly. It also shows that priming occurs in a quite similar way for music students and for listeners without any musical training or any formal knowledge of tonal music. Both results suggest that harmonic expectation is a subtle phenomenon, probably varying continuously from strong to low expectation, that is based on a quite robust cognitive process. The most important result of Experiment 3 is that global contexts alter the processing of chords, even though the immediate local contexts are identical. This demonstrates that harmonic expectancies do not only occur sequentially from chord to chord, but also depend on the harmonic function of the chord in the extended temporal context. Since a consonant/dissonant decision task forces subjects to focus on the very local information (target chord) and the information preceding the target is not explicitly helpful in this perceptual judgment, the present findings emphasize the strength of global harmonic structures in expectancy formation.

\section{GENERAL DISCUSSION}

The present experiments demonstrate that different harmonic contexts can affect the perception of the ends of musical sequences (Experiment 1) and the processing of chords, even when the immediate local context is held constant (Experiments 2-3). This confirms the importance of contextual information in music, both for the pro- cessing of chords (see Krumhansl, 1990) and for the processing of melodies (Bigand, 1997; Dowling, 1986). The present findings reveal that a previous context not only influences the understanding of the incoming events but also prepares listeners to process certain events rather than others. That these results were observed with subjects who have never practiced or learned music emphasizes the robustness of harmonic expectations driven at a global level of musical structure.

The main issue remains: to interpret this effect in the light of current models of tonal cognition. First, it can be argued that global context effects do not involve a cognitive component but simply occur at a sensory level. In our view, if sensory priming was dominant over harmonic priming, a weak or zero context effect should have been observed here, since the target and the chord that immediately preceded it were held constant. In response, it may be argued that global sensory priming (instilled by the chords preceding the penultimate one) may prevail over local sensory priming (instilled by the seventh chord). No statistical evidence was found here to support the dominance of global sensory priming. This suggests that the influence of global harmonic context may be better understood in the light of current models of tonal cognition.

Two current cognitive models might provide a different account of these global context effects. In Bharucha's (1987) neural net model, priming effects result from activation spreading via a cognitive structure that links related chords. The strength of these links expresses the internalization of the harmonic regularities pervasive in Western musical pieces. This pattern of connections constitutes a nontemporal knowledge about Western harmony that generates automatic and schematic expectations (Bharucha, 1994). In other words, priming occurs automatically inside a schematic knowledge set. On the one hand, this model provides a possible account of the present finding. A previous context activates schematic knowledge relative to a given key, which in turn facilitates the processing of the primary reference chord of the key (i.e., the tonic chord). On the other hand, it may be argued that a strict application of Bharucha's model predicts that the local activation produced by the penultimate chord for the target chord should be the same in both contexts, since their local harmonic relation is constant. The fact that the priming differs as a function of context in Experiments 2 and 3 contradicts such a prediction. Following Schmuckler and Boltz's (1994) ideas, Bharucha's connectionist model might have to explicitly incorporate temporal weighting factors in order to account for the influence of extended temporal contexts.

A similar problem was also encountered by lexical-lexical activation models of semantic priming (Foss \& Ross, 1983). In these models, priming is based on a fast acting and automatic activation that spreads in the mental lexicon via the long-term connections between items. Such a model fails to explain why semantic priming differs when words are presented in coherent sentences instead of in random lists (Gough, Alford, \& Holley-Wilcox, 1981). It also fails to explain why local semantic priming disappears 
when the global context is varied (Hess et al., 1995). One way to accommodate such global effects in connectionist models is to posit that several items in the context may be the source of priming (Duffy, Henderson, \& Morris, 1989). Activations spread automatically in the mental lexicon and are accumulated in a buffer as sentences progress. In this way, a target word may be primed differently, depending on the number of words semantically related to the target in the previous context. A similar explanation might account for the present findings: the chords defining the global context in the present sequences are more harmonically related to the target in one context than in the other. Integrating the activation pattern in working memory during the progress of the piece of music may lead to the different priming effects observed. This suggest that Bharucha's connectionist model might contain some buffer, whose size should be explicitly defined.

Connectionist models of semantic and harmonic priming propose an automatic spreading activation inside a mental lexicon (or inside a schematic musical knowledge set). In the language field, these models were challenged by discourse-based models. Discourse-based models claim that priming occurs outside the mental lexicon (i.e., above the word levels). The priming depends on the ease with which the target can be integrated in the ongoing discourse representation. Such a model recently received strong empirical support (Hess et al., 1995). In music cognition, similar discourse-based models may be found in Lerdahl and Jackendoff's (1983) and Lerdahl's (1988) tonal pitch space theory. In these models, the structural stability of the musical events depends both on their position inside the tonal hierarchy and on their temporal and rhythmic position in the context of a given piece. In other words, such music discourse-based models predict that priming would mainly depend on the way in which the target event fits in the events hierarchy, and would be weakly influenced by tonal hierarchy (see Bharucha, 1994, and Lerdahl, 1988, for a discussion of these terms). In the present study, a music discourse-based model would explain the priming effects in the following way. In one context, the targets occur after an authentic cadence that closes the structural tree. It is unexpected, since it cannot be easily integrated in a structure that is already closed. If the structural trees were not closed when the target chords occurred, weaker priming effects would probably have been observed. The purpose of the present experiment was not to disentangle these two types of models. Following Hess et al. (1995), further experiments crossing local and global harmonic contexts might address this issue.

\section{REFERENCES}

Bharucha, J. J. (1987). Music cognition and perceptual facilitation: A connectionist framework. Music Perception, 5, 1-30.

BHARUCHA, J. J. (1994). Tonality and expectation. In R. Aiello \& J. Sloboda (Eds.), Musical perceptions (pp. 213-239). Oxford: Oxford University Press.

Bharucha, J. J., \& KrumhansL, C. L. (1983). The representation of harmonic structure in music: Hierarchies of stability as a function of context. Cognition, 13, 63-102.

Bharucha, J. J., \& Stoeckig, K. (1986). Reaction time and musical expectancy: Priming of chords. Journal of Experimental Psychology: Human Perception \& Performance, 12, 403-410.

Bharucha, J. J., \& Stoeckig, K. (1987). Priming of chords: Spreading activation or overlapping frequency spectra? Perception \& Psychophysics, 41, 519-524.

BIGAND, E. (1997). Perceiving musical stability: The effect of tonal structure, rhythm, and musical expertise. Journal of Experimental Psychology: Human Perception \& Performance, 23, 808-822.

Bigand, E., Parncutt, R., \& Lerdahl, F. (1996). Perception of musical tension in short chord sequences: The influence of harmonic function, sensory dissonance, horizontal motion, and musical training. Perception \& Psychophysics, 58, 125-141.

BolTZ, M. G. (1989a). Perceiving the end: Effects of tonal relationships on melodic completion. Journal of Experimental Psychology: Human Perception \& Performance, 15, 749-761.

BolTz, M. G. (1989b). Rhythm and "good endings": Effects of temporal structure on tonality judgments. Perception \& Psychophysics, 46, 9-17.

BolTz, M. G. (1993). The generation of temporal and melodic expectancies during musical listening. Perception \& Psychophysics, 53, 585-600.

BolTZ, M. G., \& JoNES, M. R. (1986). Does rule recursion make melodies easier to reproduce? If not, what does? Cognitive Psychology, 18, 389-431.

CARLSEN, J. C. (1981). Some factors which influence melodic expectancy. Psychomusicology, 1, 12-29.

Cohen, J., MacWhinney, B., Flatt, M., \& Provost, J. (1993). PsyScope: An interactive graphic system for designing and controlling experiments in the psychology laboratory using Macintosh computers. Behavior Research Methods, Instruments, \& Computers, 25, 257-271.

CUdDY, L. L., \& LUNNEY, C. A. (1995). Expectancies generated by melodic intervals: Perceptual judgments of melodic continuity. Perception \& Psychophysics, 57, 451-462.

Dowling, W. J. (1986). Context effects on melody recognition: Scalestep versus interval representation. Music Perception, 3, 281-296.

Duffy, S. A., Henderson, J. M., \& Morris, R. K. (1989). Semantic facilitation of lexical access during sentence processing. Journal of Experimental Psychology: Learning, Memory, \& Cognition, 15, 791-801.

Foss, D. J. (1982). A discourse on semantic priming. Cognitive Psychology, 14, 590-607.

Foss, D. J., \& Ross, J. R. (1983). Great expectations: Context effects during sentence processing. In G. Flores d'Arcais \& R. J. Jarvella (Eds.), The process of language understanding (pp. 169-191). Chichester, U.K.: Wiley.

Foss, D. J., \& SPEER, S. R. (1991). Global and local context effects in sentence processing. In R. R. Hoffman \& D. S. Palermo (Eds.), Cognition and the symbolic processes: Applied and ecological perspectives (pp. 115-139). Hillsdale, NJ: Erlbaum.

Gough, P. B., Alford, J. A., \& Holley-Wilcox, P. (1981). Words and contexts. In O. L. Tzeng \& H. Singer (Eds.), Perception of print: Reading research in experimental psychology (pp. 85-102). Hillsdale, NJ: Erlbaum.

Hess, D. J., Foss, D. J., \& CARroll, P. (1995). Effects of global and local context on lexical processing during language comprehension. Journal of Experimental Psychology: General, 124, 62-82.

JONES, M. R. (1976). Time, our lost dimension: Toward a new theory of perception, attention, and memory. Psychological Review, 83, 323-355.

JONES, M. R. (1987). Dynamic pattern structure in music: Recent theory and research. Perception \& Psychophysics, 41, 621-634.

Jones, M. R., Boltz, M. [G.], \& KIDD, G. (1982). Controlled attending as a function of melodic and temporal context. Perception \& Psychophysics, 32, 211-218.

Jones, M. R., Boltz, M. G., \& Klein, J. M. (1993). Expected endings and judged duration. Memory \& Cognition, 21, 646-665.

JONES, M. R., \& YEE, W. (1993). Attending to auditory events: The role of temporal organization. In S. McAdams \& E. Bigand (Eds.), Thinking in sound: The cognitive psychology of human audition (pp. 69112). Oxford: Oxford University Press, Clarendon Press. 
KrumhansL, C. L. (1990). Cognitive foundations of musical pitch. New York: Oxford University Press.

Krumhansl, C. L. (1995). Music psychology and music theory: Problems and prospects. Music Theory Spectrum, 17, 53-81.

Lerdahl, F. (1988). Tonal pitch space. Music Perception, 5, 315-345.

LERDAHL, F., \& JACKENDOFF, R. (1983). A generative theory of tonal music. Cambridge, MA: MIT Press.

Meyer, D. E., \& Schyaneveldt, R. W. (1971). Facilitation in recognizing pairs of words: Evidence of a dependence between retrieval operations. Journal of Experimental Psychology, 90, 227-234.

Meyer, D. E., Schvaneveldt, R. W., \& Ruddy, M. G. (1974). Functions of graphemic and phonemic codes in visual word-recognition. Memory \& Cognition, 2, 309-321.

MEYER, L. B. (1956). Emotion and meaning in music. Chicago: University of Chicago Press.

MEYER, L. B. (1973). Explaining music: Essays and explorations. Chicago: University of Chicago Press.

Narmour, E. (1989). The "genetic code" of melody: Cognitive structures generated by the implication-realization model. Contemporary Music Review, 4, 45-64.

NARMOUR, E. (1990). The analysis and cognition of basic melodic structures: The implication-realization model. Chicago: University of Chicago Press.

Palmer, C., \& Krumhansl, C. L. (1987a). Independent temporal and pitch structures in determination of musical phrases. Journal of Experimental Psychology: Human Perception \& Performance, 13, 116-126.
Palmer, C., \& Krumhansl, C. L. (1987b). Pitch and temporal contributions to musical phrase perception: Effects of harmony, performance timing, and familiarity. Perception \& Psychophysics, 41, 505-518.

Parncutt, R. (1989). Harmony: A psychoacoustical approach. Berlin: Springer-Verlag.

SChELlenberG, E. G. (1996). Expectancy in melody: Test of the implication-realization model. Cognition, 58, 75-125.

SCHENKER, H. (1935). Der freie Satz. Vienna: Universal Editions.

SCHENKER, H. (1979). Free composition (E. Oster, Trans.). New York: Longman.

SCHMUCKLER, M. A. (1989). Expectation in music: Investigation of melodic and harmonic processes. Music Perception, 7, 109-150.

SCHMUCKLER, M. A. (1990). The performance of global expectations. Psychomusicology, 9, 122-147.

SCHMUCKLER, M. A., \& BolTz, M. G. (1994). Harmonic and rhythmic influences on musical expectancy. Perception \& Psychophysics, 56, 313-325.

TeKman, H. G., \& Bharucha, J. J. (1992). Time course of chord priming. Perception \& Psychophysics, 51, 33-39.

UNYK, A. M., \& CARLSEN, J. C. (1987). The influence of expectancy on melodic perception. Psychomusicology, 7, 3-23.

(Manuscript received October 11, 1995; revision accepted for publication November 4, 1996.) 